\title{
Pratiques
}

Linguistique, littérature, didactique

185-186 | 2020

Lire des documents composites en classe

\section{Le devenir littéraire}

How is literary change?

\section{Alain Viala}

\section{(2) OpenEdition}

Journals

Édition électronique

URL : http://journals.openedition.org/pratiques/8612

DOI : 10.4000/pratiques.8612

ISSN : 2425-2042

\section{Éditeur}

Centre de recherche sur les médiations (CREM)

\section{Référence électronique}

Alain Viala, « Le devenir littéraire », Pratiques [En ligne], 185-186 | 2020, mis en ligne le 30 juin 2020, consulté le 15 octobre 2020. URL : http://journals.openedition.org/pratiques/8612 ; DOI : https:// doi.org/10.4000/pratiques.8612

Ce document a été généré automatiquement le 15 octobre 2020

(c) Tous droits réservés 


\title{
Le devenir littéraire
}

\author{
How is literary change?
}

Alain Viala

1 Chaque année en France il se vend 472500 exemplaires d'ouvrages de G. de Maupassant, 425000 de Molière, 338000 de $\mathrm{V}^{\text {. Hugo }}{ }^{1}$ et on pourrait continuer avec Voltaire, C. Baudelaire, etc. : il est manifeste que les classiques de la littérature ont du succès. Sans doute cela a-t-il quelque rapport avec le fait que le rite culturel initiatique que subit chaque génération est désormais une épreuve anticipée de français (qui, au moment où je trace ces lignes fête juste ses cinquante ans) dont le caractère est indéniablement littéraire ${ }^{2}$.

2 La littérature de création n'a pas moins de succès : les dix meilleures ventes annuelles sont des romans et cette littérature caracole loin en tête des publications nouvelles. Si l'on ajoute que les instances nationales et régionales soutiennent le livre et l'art dramatique, que, comparés à d'autres, les Français plutôt grands lecteurs et plutôt assidus au théâtre - deux fois plus que les Américains par exemple ${ }^{3}$ - et enfin que la France s'enorgueillit d'être championne du monde des prix Nobel de littérature : on peut dire qu'elle continue d'être « une nation littéraire " ${ }^{4}$.

3 Et pourtant... Pourtant chaque saison voit fleurir sur les rayonnages des librairies des livres aux titres dramatiques: La Littérature en péril ${ }^{5}$, Adieu à la littérature ${ }^{6}$, Après la littérature ${ }^{7}$, Enseigner la littérature par temps mauvais ${ }^{8} .$. Et déferle un discours qui exprime, selon les termes d'A. Compagnon dans sa leçon inaugurale au Collège de France « le sentiment d'une indifférence croissante à la littérature, ou même d'une haine de la littérature $»^{9}$.

4 Ce paradoxe entre chiffres de prospérité et cris de crise mérite qu'on l'observe un peu, en ce qu'il marque la génération présente et peut engager la situation de la littérature, de son enseignement et de ceux, individus, groupes et revues qui s'y emploient. Qu'on l'observe non pour en tirer - comme druide ou augure - des prédictions, mais pour essayer de discerner comment le littéraire est devenu le lieu d'un tel paradoxe. 


\section{Le paradoxe littéraire actuel}

\section{Le discours de crise}

5 À ma connaissance, le discours de crise a été lancé par P. Sollers en 1994 dans son recueil La Guerre du goût $t^{10}$. Il est ensuite vite devenu un lieu commun, et un lieu commun au sens premier du terme puisqu'on le trouve sous la plume de très nombreux auteurs même quand ils sont en conflit les uns contre les autres (on en verra des exemples plus loin). Sans en égrener ici une liste, qui d'ailleurs ne peut être exhaustive, il suffit pour s'en faire une idée assez nette de suivre les titres que je citais à l'instant, depuis L'Adieu à la littérature de W. Marx en 2005 et La Littérature en péril de T. Todorov en 2007, jusqu'à l'Après la littérature de J. Faerber, qui date de 2018, et Enseigner la littérature par temps mauvais de B. Viard qui date de 2019, sans compter - avec quelques autres qui seront cités en temps et lieux - donc la leçon inaugurale d'A. Compagnon en 2006. À laquelle j'emprunterai encore une formule qui résume l'argument central de ces discours, à savoir que «le lieu de la littérature s'est amenuisé depuis une génération ${ }^{11} »$. C'est cet amenuisement, cette régression qui suscite le sentiment de crise. Lequel mobilise un lexique de la nostalgie, de "l'Adieu », voire de l'élégie quand le livre de J. Faerber s'ouvre par une formule d'oraison funèbre: "la littérature est morte ». Mais il mobilise aussi, quand T. Todorov proclame la littérature en danger ou que P. Sollers décrète l'état de guerre, un lexique de combat. C'est que ces auteurs convergent dans la dénonciation de quatre causes de la crise, ou plutôt de quatre coupables, les quatre cavaliers de l'Apocalypse littéraire: le numérique, dont l'irruption fait que "dans les loisirs, les écrans et le numérique morcellent le temps disponible pour les livres » (Compagnon, 2006) ; la critique, accusée de s'être avachie dans le copinage et de ne plus remplir sa mission de guide ou d'aiguillon ${ }^{12}$; le formalisme qui a fait tomber les écrivains dans une " écriture intransitive " (Faerber, 2018) qui ne produit que des « objets langagiers d'une conception étriquée et coupée du monde " (Todorov, 2007); et enfin, le plus blême des quatre, l'École, l'institution scolaire et universitaire, accusée de ne pas donner assez de place de la littérature et surtout d'avoir propagé le virus des théories formalistes inoculées par la trinité structuraliste W. Marx/F. de Saussure/S. Freud selon P. Sollers et T. Todorov, ou, selon B. Viard, le trio post-structuraliste R. Barthes/M. Foucault/P. Bourdieu. Ainsi nimbé de nostalgie rageuse, ce discours relève bien d'un « sentiment de crise ».

Pour une analyse objective, un sentiment qui passe en lieu commun constitue une donnée au même titre, quoique d'une autre nature, que les événements ou les statistiques. Donc il ne s'agit pas de savoir si ce discours a, ou non, raison, mais d'en voir les raisons.

\section{Une crise de croissance}

7 Pour tenter d'objectiver la subjectivité de ce « sentiment de crise», on peut convoquer - avec les précautions voulues -, les données chiffrées afin de déchirer le brouillard des impressions ${ }^{13}$, il apparaît qu'il y a bien une crise, mais en fait, une crise de croissance.

Dans les pratiques d'écriture, d'édition et de critique, il est manifeste que le champ littéraire connaît une surabondance de productions. Ainsi chaque rentrée voit paraître de plus de 500 romans, dont le Syndicat national de l'édition souligne que très peu sont 
de "de longue vente », c'est-à-dire restent plus d'un an en librairie ${ }^{14}$. L'édition jette sans cesse sur le marché de nouveaux titres qui n'y restent que le temps d'un " dépôt d'office ", soit trois mois. C'est que la courbe du chiffre d'affaires est à la stagnation, tandis que celle du nombre de titres est en hausse et celle du temps moyen de présence en rayon à la baisse ${ }^{15}$. D'où un "effet de ciseaux", typique d'une crise de surproduction.

9 La littérature a l'habitude de telles crises : on se souvient des Illusions perdues ou encore de la crise naturaliste étudiée par C. Charle ${ }^{16}$. Mais depuis les années 1980, la globalisation, la hausse du prix du papier et la concurrence des médias à écrans ont imposé aux maisons d'édition de s'intégrer à de grands groupes d'industries culturelles ${ }^{17}$. Le modèle économique de l'édition tel qu'il s'était forgé au xix ${ }^{e}$ siècle est désormais balayé ${ }^{18}$. Les capitalistes de ces conglomérats exigent des retours rapides sur investissement et regardent la littérature comme un produit parmi d'autres; d'où la multiplication des titres et leur rotation plus accélérée que jamais pour dégager du profit en multipliant l'offre face à une demande atone. D'où aussi une monoculture du roman, car la poésie et les textes de théâtre se vendent peu. Et d'où, enfin et évidemment, un primat de la valeur marchande au détriment de la valeur esthétique.

10 Aussi, cette évolution du marché est liée à la croissance des lectorats et de la formation qu'ils ont reçue, donc au rôle de l'École. Laquelle, on le sait, a connu une mutation sur un siècle, puisque que le Baccalauréat, le diplôme qui concernait il y a cent ans, $1 \%$ d'une classe d'âge est aujourd'hui le lot de $80 \%$. Et cette mutation a connu une accélération en une génération, puisqu'on est passé de 270000 bacheliers en 1985 à 700000 aujourd'hui (en chiffres arrondis). Dans cette démocratisation, la référence littéraire est devenue une base vraiment commune de la culture légitime, comme le traduisent l'épreuve anticipée de français et les ventes des "classiques». Mais à l'inverse la série littéraire qui représentait $75 \%$ des bacheliers au début du $\mathrm{xx}^{\mathrm{e}}$ siècle, était tombée à $50 \%$ en 1960 et a aujourd'hui dégringolé à $8 \%$. Autre cas de « courbes en ciseaux ", autre indice d'une perte de prestige.

11 Le "sentiment d'une indifférence croissante à la Littérature» (Compagnon, 2006) traduit donc cette conjonction entre la perte du prestige esthétique sur le marché marchand et celle du prestige intellectuel sur le marché scolaire. De fait, ceux qui l'expriment sont des critiques et des enseignants, donc des professionnels du commentaire: comme leur position sociale repose sur la valeur symbolique de la littérature, leurs prises de positions naissent de la baisse de cette valeur sur le marché des biens symboliques, signe, si besoin était, que les façons de penser ne se jouent pas dans le ciel empyrée des idées pures. Non que ces enjeux de valeurs ne soient de mises, mais il est fréquent que les discours polémiques se trompent d'ordres, et du coup trompent leurs audiences ${ }^{19}$. Notamment en matière d'idées esthétiques et de théories de la littérature.

\section{Le brouhaha et la guerre au formalisme}

\section{Le brouhaha esthétique}

Dans le champ littéraire aujourd'hui, le tsunami éditorial charrie tant de choses diverses que les manières d'écrire se dispersent et les thématiques s'enchevêtrent. À un point tel que la production actuelle induit, pour reprendre un terme de L. Ruffel, un 
effet de «brouhaha $»^{20}$. Un indicateur tel que le prix Goncourt en témoigne: il est parfois attribué à des encore débutants (E. Vuillard, L. Slimani) mais aussi à des très chevronnés (J. Echenoz, P. Quignard, M. Houllebecq), et en tout cas à des auteurs dont les choix thématiques et formels ne dessinent pas un fil conducteur mais un enchevêtrement.

Cependant, quand des critiques essayent pourtant de discerner les voix de ce brouhaha, tous, de D. Viart à J. Faerber en passant par A. Compagnon et T. Todorov, s'échinent à classer et hiérarchiser pour désigner ce qui mérite à leurs yeux d'être vraiment appelé «littérature ». Ainsi D. Viart distingue-t-il la littérature «consentante " (traditionnelle), la « concertante » (faussement moderne) et la " déconcertante », seule valide à ses yeux $^{21}$. J. Faerber distingue pour sa part les «incontemporains", les «mécontemporains» (faussement provocateurs, comme M. Houllebecq) et les (vrais) «contemporains». Surtout, tous rebutent les productions populaires, policières, multimédiatiques et proprement «engagées ». Réflexe de la critique à la française : là où les anglo-saxons mettent en avant des thématiques, notamment celles de l'écriture post-coloniale et de l'écriture féminine, qui jouent un rôle de premier plan dans la production en langue française depuis une génération, les critiques français insistent tous sur le fait que depuis 1980 la littérature est marquée par la recherche, comme un moyen de préserver le prestige esthétique en période de surproduction, de formes narratives très soignées.

Et la plupart convergent pour déplorer cette prépondérance de la forme. W. Marx y voit un effet de «l'adieu à la littérature », J. Faerber qualifie J. Echenoz et P. Michon de «statues du commandeur» d'aujourd'hui ${ }^{22}$, et A.Compagnon - que par ailleurs J. Faerber cloue au pilori - affirme qu'il s'agit là de la conséquence des options prise par les «avant-gardes du Nouveau Théâtre et du Nouveau Roman, puis de l'écriture minimaliste [qui] ont visé à réduire le médium [littéraire] à son essence $»^{23}$. Bref, lieu commun à l'intérieur du lieu commun de crise, se ressasse ainsi l'idée (selon la formule de Todorov, 2007) d'une «conception de la littérature comme un objet langagier autosuffisant ».

15 Cette thèse du formalisme esthétique est au cœur du discours de crise dans sa dimension combattive, polémique, qui constitue un des points névralgiques de la crise, en ce qu'elle fait passer le débat du champ littéraire au champ didactique.

\section{L'éventail théorique}

Du côté des théories de la littérature, la situation est en partie similaire et en partie différente, selon l'histoire propre de ces théories. Je suppose l'histoire de la théorisation littéraire assez familière aux lectrices et lecteurs de ces pages pour que l'on puisse se contenter de brefs rappels. Le premier concerne l'essor, dans les années 1960-1970, d'une mouvance des approches structurales, où la linguistique, principalement, mais aussi la pragmatique et la narratologie offraient à l'évidence des outils pertinents pour l'analyse des textes. On sait qu'une revue comme Pratiques a été fondée, en 1974, avec le souci de rendre les avancées de la recherche en sciences du langage disponibles et opératoires pour les enseignants.

17 Mais on sait aussi qu'une des branches de cette mouvance a soutenu, dans la lignée des Formalistes russes, l'hypothèse d'une propriété intrinsèque de la littérature, la littérarité. Elle prenait appui sur la linguistique structurale : c'est ainsi que T. Todorov 
envisageait les textes comme des «paroles » mettant en œuvre une « langue » littéraire dont il s'agirait de définir les structures. Mais cette branche a dû, on le sait, dresser le bilan, transcrit en 1991 par G. Genette dans Fiction et diction ${ }^{24}$, qu'il n'est de littérarité que relativement à des situations d'écriture et de lecture.

Or au même moment se développaient des préoccupations touchant notamment aux Implications sociales des textes. En particulier, tout un courant de sociocritique ${ }^{25}$. Mais aussi une approche plus proprement sociologique, dont on peut prendre pour repère Les Règles de l'art. Genèse et structure du champ littéraire de P. Bourdieu, publié en $1992^{26}$.

Cet ouvrage s'inscrit dans l'héritage des sciences de référence de l'analyse structurale chez C. Lévi-Strauss, les sciences de l'homme et de la société. Et il met en lumière comment le champ littéraire est un lieu de luttes pour la définition légitime de la littérature. Donc une relativité intrinsèque de l'objet.

Plus largement, il s'est produit depuis ces années-là un regain des propositions théoriques ${ }^{27}$ et la palette s'est élargie à, outre la sociologie du champ et l'histoire du fait littéraires, la sociocritique ${ }^{28}$, l'ethnocritique ${ }^{29}$, la sociopoétique ${ }^{30}$, des reprises de la mythocritique, etc. Ces propositions témoignent, de diverses façons, d'un souci d'inscrire ce qu'on appelle « littérature » dans des ensembles de pratiques englobantes.

Cet abrégé historique appelle deux observations. L'une étant, que si l'éventail des théories critiques s'est ouvert ces dernières années, il ne s'est pas pour autant dispersé comme grenaille à l'instant de l'explosion, même s'il ne constitue pas un ensemble structuré. L'autre observation concerne plus précisément la diversité des propositions, et le fait qu'elle soit ou non reconnue comme telle, ce qui n'est pas toujours le cas.

\section{L'accusation formaliste et la lecture immanente}

En effet, alors que l'état des pratiques fait apparaître que, depuis trente ans, les enseignants et chercheurs qui s'attachent à expliciter les théories avec lesquelles ils travaillent ne revendiquent pas le formalisme, voire en remettent en cause l'idée centrale, celle de « l'objet langagier clos sur lui-même ", l'accusation de formalisme est ressassée dans les discours de crise. C'est donc qu'elle ne fonctionne pas tant comme une description de la crise mais comme un argument polémique, comme un essai pour incriminer des coupables.

C'est sans doute T. Todorov qui a formulé le plus nettement cette accusation contre le formalisme. Il souligne qu'après avoir envahi le champ littéraire il se serait répandu dans le champ didactique : " cette conception étriquée de la littérature, qui la coupe du monde dans lequel on vit, s'est imposée dans l'enseignement ». A. Compagnon soutient la même idée et ironise sur le fait que le roman le plus détesté des Français, c'est Madame Bovary, parce qu'il a été étudié en classe (ibid.). Bref, le formalisme aurait stérilisé l'écriture puis stérilisé l'enseignement. Ce serait la cause majeure de l'«l'indifférence croissante à la littérature", et les tenants du discours de crise dessinent ainsi deux camps : d'un côté, des formalistes qui, disent-ils en substance, en faisant des lectures médiées par des théories enseignent en fait ces théories et non la littérature elle-même ; et de l'autre, eux qui se posent en défenseurs celle-ci.

Ils proposent, eux, de promouvoir un contact direct avec les textes, une lecture immanente, seule capable de donner accès aux «leçons de vie » (Todorov, 2007 ; voir aussi Compagnon, 2006, § 24) que porte la littérature. Cette lecture immanente apparaît 
comme une héritière banalisée du « jugement sans concept » kantien. Elle s'inscrit dans une philosophie du singulier universel: les écrits des grands auteurs incarneraient mieux que tout l'expérience singulière digne d'être élevée en expérience valide pour tous. Ces grands auteurs porteurs de leçons de vie méritent admiration et obédience ; ce dont leurs commentateurs attitrés peuvent escompter en bénéficier à leur tour, par délégation en quelque sorte; on retrouve là les enjeux de la quête de prestige. Enfin, définir de la sorte la littérature, sa raison d'être et les modalités de son étude constitue évidemment une théorie au même titre que les autres, mais qui, pour mieux combattre les autres, ne s'avoue pas comme telle, vieille tactique de toutes les querelles. Mais là encore les idées ne sont pas des alcyons qui planeraient dans un ciel éthéré, et ces oppositions retraduisent en fait des contradictions inscrites dans des pratiques liées à l'évolution du champ didactique.

\section{L'évolution du champ didactique des Lettres}

L'histoire du champ scolaire mériterait sans nul doute une étude détaillée. Dans les limites du présent propos, il suffira de résumer ce qui concerne le domaine littéraire.

\section{Ouverture et spécialisation}

Dans les universités, globalement, la croissance du nombre d'élèves du Secondaire a induit à partir des années 1960 une forte croissance du nombre d'étudiants. En particulier - quoique pas seulement - dans les secteurs qui forment les futurs enseignants, on va en voir une conséquence. Car, là aussi, cette croissance d'ensemble a produit des répartitions différentes selon les filières et les finalités. Pour le domaine des Lettres, on peut repérer une première phase de diversification lors de l'accélération de la démographie scolaire et universitaire des années 1980. Les Unités d'Enseignement et de Recherche (UER, tel était le nom à l'époque) de Lettres portaient alors en leur sein des options théâtre, cinéma et communication. Ainsi se posait la question, très concrète de ce que pouvait être la part de formation commune à ces différents usages du langage. Ce pourquoi les démarches inspirées des sciences humaines et sociales pouvaient rendre de bons services. Mais en pratique, ces options se sont alors assez vite autonomisées en départements de plein exercice: la diversification est devenue scission.

En partie de leur fait, et en partie par le souhait des littéraires. C'est que, pour ces derniers, le besoin de professeurs du Secondaire faisait augmenter les postes mis aux concours et donc les effectifs étudiants des formations correspondantes. De ce fait, les départements de littérature ont privilégié ces formations spécialisées dans la préparation aux concours d'enseignement, et s'est exercée en particulier l'influence du modèle du plus prestigieux, d'entre eux, l'agrégation: donc l'histoire littéraire nationale des grands écrivains. Les tenants des lectures immanentes ont trouvé là un terrain propice.

Mais dans un deuxième temps, une fois que la courbe du nombre de bacheliers ne pouvait plus grimper au même rythme, les postes mis aux concours ont stagné et avec eux les effectifs d'étudiants concernés. Ainsi, en moins de vingt ans, de 1995 à 2013, les premiers cycles universitaires de Lettres sont tombés de 68835 étudiants à 65 598, et si 
les chiffre semblent peu différents, il faut les pondérer par les pourcentages qu'ils représentent :

- 9,56 \% du total des étudiants en 1995 ;

• 7,27 \% en 2013. imposé le besoin d'ajuster les programmes du Secondaire. Le Baccalauréat devenait le seuil commun, normal, de formation. Dès lors, la discipline appelée "français " ne visait pas à préparer à des études de Lettres mais à doter les bacheliers d'un bagage pertinent quelle que soit leur filière. Les Lettres s'affirmaient comme la "discipline cruciale " $^{32}$. Chacun sait quel conflit s'est dessiné à ce sujet depuis l'an 2000. D'un côté ont été formulées des propositions de programmes qui visaient à doter tous les élèves de moyens d'analyse et d'expression. À cette fin, elles recouraient selon que de besoin à des outils pris, pour les analyses de formes, dans la pragmatique, la narratologie et la poétique et, pour celle des contenus, dans l'histoire, l'anthropologie et la sociologie. Bien évidemment, le corpus et l'histoire littéraire étaient français. Et s'opérait même un " recentrage littéraire ${ }^{33}$. Mais ont alors surgi des protestations argumentées par le souci sur des « leçons de vie ». Il est apparu qu'elles correspondaient aussi au désir de 
cultiver ce «noble tronc » littéraire qui porte les fruits de «l'esprit de la nation $»^{34}$. Du pragmatisme d'un côté, une option idéologique de l'autre.

Et les ennemis des théories recourent à un vocabulaire de mobilisation (T. Todorov dit " péril », P. Sollers dit " guerre » et A. Compagnon parle de " défense) qui glisse parfois au lexique du forfait de trahison, comme dans le livre d'A. Joste, Contre-expertise d'une trahison $^{35}$. Il va même aujourd'hui jusqu'à l'accusation d'assassinat, quand C. Barjon demande Mais qui sont les assassins de l'École ? ${ }^{36}$ ou que J.-P. Brighelli affirme que C'est le français qu'on assassine; selon lui le crime profite à la mondialisation, pour laquelle « l'objectif, c'est que la France doit disparaître, et c'est en attaquant à la base sa langue et sa culture qu'on en finira le plus vite avec elle $»^{37}$. Un tel paroxysme de propos au vitriol fait, en lui-même, crise. Il demande aussi d'observer ce qu'il engage.

\section{Affaires de lecture.}

Comme il se doit quand il s'agit d'enseigner la littérature, des oppositions se manifestent, en particulier dans les pratiques de lecture proposées aux élèves. Les programmes de lycée mis en place en 1999-2000 ayant opéré un recentrage sur la littérature, ce n'est pas tant le corpus concerné qui fait débat que les modalités supposées requises. Et là se confirme le paradoxe du "sentiment de crise ». En effet, les combats ne portent pas tant sur des questions proprement pédagogiques, telles que les conditions concrètes de la lecture (lire des extraits ou des œuvres complètes, notamment) que sur les finalités. Non pas tant la promotion de la lecture et l'acquisition d'un savoir-lire à finalité utilitaire que tout le monde s'accorde à promouvoir, que le but de cette acquisition. L'histoire de l'enseignement littéraire depuis une génération ${ }^{38}$ fait apparaître que si les années 1980 ont vu une poussée de la finalité qu'on peut appeler cognitivo-langagière, autrement dit une traduction en pratique de la thèse de la littérarité, depuis la fin des années 1990 s'observe une forte hausse d'une finalité «sociale et philosophique $»^{39}$. Et dans ce cadre s'est développé ces dernières années plus que jamais - un discours sur le «sujet lecteur $»^{40}$. Il traduit les postulations des tenants de la lecture immanente. Y compris sous les plumes de représentants de l'institution, il ressasse le désir d'«échapper aux démons du formalisme $»^{41}$ : on voit bien que celui-ci ayant été depuis longtemps abandonné, y compris par ses promoteurs, c'est peindre le diable sur la muraille. Mais la suite des mêmes discours révèle pourquoi : il s'agit de faire place à " la dimension subjective de la lecture ", et de "recréer un rapport heureux à celle-ci quelle que soit l'hétérogénéité culturelle, sociale et cognitive des élèves ${ }^{42}$.

Ainsi apparaît ce qui est au cœur de la "crise »: le désir d'inculquer un modèle de comportement où l'individu (le "subjectif») est requis d'aimer lire. Il ne s'agit alors pas seulement d'acquérir un "savoir-lire », sans préjuger des investissements qu'il en fera et en tenant compte des différences de classes et donc de capital culturel incorporé en habitus afin de les dépasser, mais bien en vue d'une communauté de vision.

De la sorte, quand la crise s'est déplacée du champ littéraire au champ de l'École et, dans ce glissement s'est aigrie en querelle, elle révèle que l'enjeu n'est pas tant la littérature que ses usages. Et c'est là qu'apparaît une évidence tellement évidente qu'on risquerait, comme la lettre volée d'Edgar-Allan Poe, de ne plus la voir. 


\section{En guise de conclusion : la qualification littéraire} qui sont eux-mêmes des opérations de qualification. En effet, par les sujets qu'ils choisissent, par les genres et registres dans lesquels ils les inscrivent, et par les modalités énonciatives selon lesquelles ils les proposent, ils ne cessent de classer et juger, même et surtout de façon implicite. Le littéraire ${ }^{44}$ apparaît donc comme un espace de qualifications à plusieurs étages : parmi tous les textes qui qualifient, et de plusieurs façons, certains ont qualifiés comme « littérature » et le façon de les aborder et d'y adhérer ou non constitue à son tour un élément de la qualification culturelle et donc de l'intégration sociale. Si on le regarde sans dogme, le littéraire participe ainsi, manifestement, aux opérations générales de qualification qui font le tissu de la sociabilité ${ }^{45}$. Car, dès lors qu'elle n'est pas purement descriptive, la qualification constitue un jugement, donc une construction de valeurs. Le constat d'échec des idées absolues de la littérature, tant dans la littérarité formelle que dans les «leçons de vie ", révèle les enjeux de ces jeux de qualification : là s'édifient les modalités des possibles adhésions des destinataires supposés par les textes. Là se joue, dans l'hypothèse immanentiste, la lecture "heureuse » quand le lecteur réel coïncide avec le lecteur supposé et jouit d'adhérer au jeu; ou, dans les perspectives qui théorisent explicitement, la construction des capacités critiques, de l'intelligence culturelle qui naît de la problématisation même de ces processus.

Revenons au mot de "crise ". Il appartient au vocabulaire médical, où il désigne un moment décisif. Alors, si de la crise actuelle nul, sauf s'il est druide ou augure, ne peut dire l'issue, il faut voir ce qu'elle a d'ores et déjà fait ressortir. Or, outre les conflits, elle fait voir l'éventail des théories littéraires aujourd'hui présentes. Et elle permet de constater qu'aucune ne couvre tous les échelons de l'opération qualifiante; au plus, chacune en analyse un ou quelques niveaux ou aspects. S'impose ainsi l'évidence qu'une réflexion qui s'attacherait à éclairer quelle place ou quelles places chacune peut occuper dans l'approche du littéraire serait sans nul doute salutaire. Et une revue qui suit depuis une génération les propositions qui adviennent en matière de recherche et leurs devenirs, si elle s'attachait à un tel inventaire et une telle analyse serait utile; et même nécessaire ; en tout cas : pratique ${ }^{46}$. 


\section{BIBLIOGRAPHIE}

ANGENOT, M. (1889). Un État du discours social. Montréal : Éditions Balzac.

AïssAouı, M. (2012). « Le top 50 des auteurs classiques les plus vendus ». Le Figaro. Mise en ligne le 14 mars 2012. En ligne : https://www.lefigaro.fr/livres/

2012/03/14/03005-20120314ARTFIG00604-le-top-50-des-auteurs-classiques-les-plus-vendus.php.

ANDRÉ, M.-O. et al. (2011), « La littérature française contemporaine à l'épreuve du fichier central des thèses ». Revue d'histoire littéraire de la France 111, pp. 695-716. En ligne : https:// www.cairn.info/revue-d-histoire-litteraire-de-la-france-2011-3-page-695.htm.

BARJON, C. (2016). Mais qui sont les assassins de l'école ?. Paris : R. Laffont

BEAUTHIER, S., DE LISLE, I. \& LE GRANDIE, E. (2018). Annales Bac 2018 Français $1^{\text {eres }}$. Paris : Hachette.

BoIssinot, A. (2016), « Les programmes officiels ». In : Petitjean, A. (dir.). Didactique du français et de la littérature. Metz : Université de Lorraine. En ligne : http://journals.openedition.org/lidil/4711.

BOURDIEU, P., (1992). Les Règles de l'art, genèse et structure du champ littéraire. Paris : Éditions du Seuil. Bourdieu, P., ChAmboredon, J.-C. \& PASSERon J.-C. (2005) [1968]. Le Métier de sociologue. Préalables épistémologiques. Berlin/Paris/La Haye : M. De Gruyter.

BRIGHELLI, J.-P. (2017). C'est le français qu'on assassine. Paris : Éd. Blanche.

CHARLE, C. (1979). La Crise littéraire à l'époque naturaliste. Roman, théâtre et politique, essai d'histoire sociale des groupes et des genres littéraires. Paris : Presses de l'École normale supérieure.

CHARTIER, R. \& MARTIN, H.-J. (1982) L'Histoire de l'édition. Paris : Promodis.

CHRISTIN, A. \& DONNAT, O. (2014). « Pratiques culturelles en France et aux États-Unis, éléments de comparaison 1981-2008 »Cultures études 2014-1. En ligne : https://www.culture.gouv.fr/Sitesthematiques/Etudes-et-statistiques/Publications/Collections-de-synthese/Cultureetudes-2007-2020/Pratiques-culturelles-en-France-et-aux-Etats-Unis.-Elements-decomparaison-1981-2008-CE-2014-1.

CNOCKAERT, V., PRIVAT, J-M. \& SCARPA, M. (éds) (2011). L'Ethnocritique de la littérature. Montréal : Presses de l'Université du Québec.

COMPAGNON, A. (2006). La Littérature, pour quoi faire? Leçon inaugurale prononcée le jeudi 30 novembre 2006. Paris : Collège de France/Fayard. En ligne : https://books.openedition.org/cdf/524.

DOMECQ, J-P. (2002)., Qui a peur de la littérature ?. Paris : Mille et une nuits.

DUCHET, C. et al. (1979). Sociocritique. Paris : Nathan.

DUFAYS, J. \& BRUNEL, M, (2016), « la didactique de la lecture et de la littérature », In : Petitjean, A. (dir.). Didactique du français et de la littérature. Metz : Université de Lorraine, p. 233-266.

FAERBER, J. (2018). Après la littérature. Écrire le contemporain. Paris : Presses universitaires de France. FRAGONARD, M.-M., GUYOT S. \& ROUSSILLON, M. (2018). Littéraire. Pour Alain Viala. Arras : Artois presses université.

GENETTE, G. (2004) [1991]. Fiction et diction. Paris : Éditions du Seuil. 
JOSTE, A. (2002). Contre-expertise d'une trahison. La réforme du français au lycée. Paris : Mille et Une Nuits.

JOURDE, P. (2002). La Littérature sans estomac. Paris : L'Esprit des péninsules.

Louichon, B. (2016). « Dix ans de sujet lecteur », In : Petitjean, A. (dir.). Didactique du français et de la littérature. Metz : Université de Lorraine.

MARX, W. (2005). L'Adieu à la littérature. Histoire d'une dévalorisation VXIII-XX siècle. Paris : Les Éditions de Minuit.

MOLLIER, J.-Y. (2015). Une autre histoire de l'édition française. Paris : La Fabrique.

MONTANDON, A (2016). « Sociopoétique ». Sociopoétique 1. En ligne : http://sociopoetiques.univbpclermont.fr/mythes-contes-et-sociopoetique/sociopoetiques/sociopoetique.

PARKHURST FERGUSON, P., (1991) [1987]. La France nation littéraire. Trad. de l'anglais par R. Rosi. Bruxelles : Éd. Labor.

PETITJEAN, A. (2014) « 40 ans d'histoire de la "lecture littéraire" au secondaire à partir de la revue Pratiques ». Pratiques 161-162. En ligne : https://journals.openedition.org/pratiques/2155.

PETITJEAn, A. (dir.) (2016). Didactique du français et de la littérature. Metz : université de Lorraine.

POPovic, P. (2011), « La sociocritique. Définition, histoire, concepts, voies d'avenir », Pratiques 151-152, p. 7-38. En ligne : https://journals.openedition.org/pratiques/1762.

Projet ANR Agon (2012). Projet Agon. La dispute : cas, querelles, controverses et création à l'époque moderne. En ligne : http://www.agon.paris-sorbonne.fr/fr.

RICHARD, S. (2004). Finalités de l'enseignement de la littérature et de la lecture des textes littéraires au secondaire. Thèse de doctorat en didactique : Université de Laval.

ROBIN, R. (1984). « Les discours social et ses usages ». Cahiers de recherches sociologiques 2 (1), p. 5-17. En ligne : https://www.erudit.org/fr/revues/crs/1984-v2-n1-crs1515818/1001976ar/.

RowE, A. (2019) « 7 Publishing Insights Revealed By Last Year's Top 100 Bestselling Books ». Forbes. Mise en ligne le 5 janv. 2019. En ligne : https://www.forbes.com/sites/adamrowe1/2019/01/05/ publishing-insights-revealed-by-last-years-top-100-bestselling-books/\#2f8701166913.

RUFFEL, L. (2016). Brouhaha. Lagrasse : Verdier.solleRs, P., (1996) [1994]. La Guerre du goût. Paris : Gallimard.

Syndicat national de l'édition (2017), L'édition en perspective, 2016-2017. Rapport d'activité du syndicat national de l'édition. Paris : Syndicat national de l'édition. En ligne : https://www.sne.fr/app/ uploads/2017/10/Edition-en-perspective-2017_Rapport-d-activite_SNE-1.pdf.

Syndicat national de l'édition (2018). Les chiffres de l'édition, 2017-2018. Rapport statistique du SNE. Paris : Syndicat national de l'édition. En ligne : https://www.sne.fr/app/uploads/2018/07/ RS18_BatWEBSignet-1.pdf.

TODOROV, T. (2007). La Littérature en péril. Paris : Flammarion.

VIART, D. (2013). «L'histoire littéraire du contemporain ». Tangences 102, p. 113-130. En ligne : https://doi.org/10.7202/1022660ar.

VIARD, B. (2019). Enseigner la littérature par temps mauvais. Lormont : Le Bord de l'Eau. 
VIALA, A. (2000). « Les Lettres, une discipline plus cruciale que jamais ». Le Monde. Mise en ligne le 22 mars 2000. En ligne : https://www.lemonde.fr/archives/article/2000/03/22/les-lettresdiscipline-plus-cruciale-que-jamais_3687359_1819218.html.

VIALA, A. (2015) « Le canon caché », In : Bishop, M.-F. \& Belladjin, A. (éds). Les patrimoines littéraires à l'école. Tensions et débats actuels. Paris : Champion, p. 83-92.

ZIMA, P. (1985). Manuel de sociocritique. Paris : Picard.

\section{NOTES}

1. Aïssaoui (2012).

2. Il suffit pour le constater d'inventorier, par exemple, les sujets de l'épreuve anticipée de français 2018 (Beauthier, de Lisle \& Le Grandic, 2018). On attend de voir ce que deviendra cette épreuve et ce que sera le "grand oral » annoncé dans les réformes en cours à ce jour.

3. Christin \& Donnat (2014). La comparaison avec d'autres pays est rendue délicate par le découpage même des catégories: ainsi le Royaume Uni n'utilise pas la catégorie "Littérature ", mais celles de "Fiction ", "Non-fiction » et de "Crime and Thrillers ». Si l'on prend en compte les «Fiction» et "Crimes and Thrillers", on retrouve des proportions équivalentes à celle de la «Littérature» en France, voir un peu supérieures. Voir Rowe (2019).

4. Parkhurst Ferguson (1991) [1987].

5. Todorov (2007).

6. $\operatorname{Marx}(2005)$.

7. Faerber (2018).

8. Viard (2019).

9. Compagnon $(2006, \S 27)$

10. Sollers (1996 [1994], Introduction).

11. Compagnon $(2006, \S 25)$.

12. Voir notamment Jourde (2002).

13. Chacun sait que c'est l'idée directrice de l'utilisation des statistiques formulée dans Le Métier de sociologue. Préalables épistémologiques (Bourdieu, Chamboredon \& Passeron, 2005 [1968]).

14. Syndicat national de l'édition (2017; le cas de "longue vente » cité est celui du roman de L. Slimani, Chanson douce, prix Goncourt de 2016).

15. Syndicat national de l'édition (2018).

16. Charle (1979).

17. Ainsi, à titre d'exemple, la maison Hachette - Figure typique du capitalisme éditorial familial du XIX ${ }^{\mathrm{e}}$ siècle - a été, après le retrait du dernier héritier de la famille, rachetée par Lagardère. S'est alors enclenché un processus d'expansion à l'international en même temps que se continuait le rachat d'autres maisons en France (A. Colin, Fayard, Grasset, Larousse, Stock, etc.). 
18. Voir, outre L'Histoire de l'édition (Chartier \& Martin, 1982), Une autre histoire de l'édition française de J.-Y. Mollier (2015).

19. Voir Projet ANR Agon (2012).

20. Ruffel (2016).

21. Je reprends ses catégories selon la version commodément lisible qu'il en donne dans Tangence $\mathrm{n}^{\circ} 102$ (Viart, 2013).

22. Et J.-P. Domecq (2002) aussi bien que P. Jourde (2002) critiquent également l'art formel « désinvolte » de J. Echenoz.

23. «La littérature a été emportée par le projet d'aller toujours plus loin, suivant un élan qui prit avec les avant-gardes la forme du «toujours moins »: purification du roman et de la poésie, concentration de chaque genre sur lui-même, réduction de chaque médium à son essence. Les défis techniques occupaient le premier plan: restriction du personnage au point de vue ou au monologue intérieur, puis effacement du personnage. Le Nouveau Roman se dressait contre le roman d'analyse, la poésie contre le récit, le Texte contre l'auteur... On ne regardait pas en arrière ni sur le côté, le bas-côté de l'autre littérature, la «littérature de boulevard", celle qui se lit. Toute mention du pouvoir de la littérature était jugée obscène, car il était entendu que la littérature ne servait à rien et que seule comptait sa maitrise d'elle-même ». Compagnon $(2006, \S 31)$

24. Genette (2004 [1991], réed. avec Introduction à l'architexte).

25. Voir notamment Duchet et al. (1979) et Zima (1985). Voir aussi, en synthèse, P. Popovic, (2011). On peut compléter avec les travaux de M. Angenot et de R. Robin sur le « discours social».

26. Bourdieu (1992).

27. Notamment les travaux du Groupe de Recherches Interdisciplinaires sur l'Histoire $\mathrm{du}$ Littéraire (voir le site https://www.ehess.fr/fr/groupe-recherchesinterdisciplinaires-sur-lhistoire-littéraire-grihl). Voir aussi Littéraire. Pour Alain Viala (Fragonard, Guyot \& Roussillon, 2018).

28. https://doi.org/10.4000/pratiques.1762 (dont la synthèse citée dressée en 2011 par P. Popovic).

29. Cnockaert, Privat \& Scarpa (2011).

30. Sociopoétiques (http://revues-msh.uca.fr/sociopoetiques/), dont A. Montandon (2016).

31. André et al. (2011).

32. Je me permets de renvoyer à Viala (2000).

33. «L'abandon du résumé-discussion ainsi que la définition par les programmes des nouveaux objets d'étude manifestent en fait, au début des années 2000, un évident recentrage sur le littéraire, recentrage qui s'est confirmé depuis à l'inverse de ce que prétendaient certains polémistes ». (Boissinot, 2016, p. 51)

34. Compagnon (2006, § 24).

35. Joste (2002).

36. Barjon (2016).

37. " "La France, ton français fout l'camp !" : baisse dramatique du niveau orthographique, conséquence d'un enseignement à la dérive ; réformes absurdes de 
l'apprentissage de la langue, "négociations" en classe sur la graphie et "simplification" de la grammaire - ; jusqu'à ce qu'il n'en reste rien ; utilisation massive de l'anglais - et, pire, du globish - par de hautes instances françaises, y compris des candidats à la présidence de la République... Tout révèle une inspiration commune : la langue, c'est la Nation, et dans le grand concert mondialisé, les nations ne sont pas bienvenues. Du coup, les "communautés" s'organisent autour de leurs propres langages, et y sont souvent encouragées par l'institution éducative : l'arabe ou le turc oui, mais le français, de moins en moins. Si la langue française est la base de notre culture, la dégrader peut suffire à suicider une France vieille de douze siècles. Mais c'est cela aussi, l'objectif : la France doit disparaître, et c'est en attaquant à la base sa langue et sa culture qu'on en finira le plus vite avec elle. » (Brighelli, 2017)

38. Voir Petitjean (2014).

39. Dufays \& Brunel (2016, p. 236). Les auteurs s'appuient en particulier sur la thèse de Richard (2004).

40. Louichon (2016, p. 387).

41. Louichon (2016, p. 390, 394).

42. Louichon (2016).

43. Voir Viala (2015).

44. Nous employons ce terme pour éviter les pièges inhérents à l'emploi de celui de " littérature ", d'autant que celui-ci est en fin de compte historiquement marqué et, sur l'échelle de la longue durée, assez récent.

45. Je me permets de renvoyer ici à Viala (2019), qui étudie comment s'est opérée au fil de l'histoire - y compris de manières contradictoires - la qualification galante comme attribution ou dénégation de valeurs sociales, éthiques, esthétiques; et "La côte d'Adam, la cuisse de Marcel et les deux bouffons de Molière. Pour une sociopoétique de la qualification ", (Sociopoétiques en ligne : (http://sociopoetiques.univ-bpclermont.fr).

46. Voici quelques revues clés permettant d'appuyer les propos soutenus au cours de l'article: Poétique, fondée en 1970, par G. Genette et T. Todorov (https:// www.cairn.info/revue-poetique.htm); Pratiques fondée en 1974 (https:// journals.openedition.org/pratiques/) ; Sociopoétiques (http://revues-msh.uca.fr/ sociopoetiques/).

\section{RÉSUMÉS}

Le début $\mathrm{du} \mathrm{xxI}^{\mathrm{e}}$ siècle est marqué par une persistante "crise de la littérature ». La chose n'est pas neuve en elle-même : la littérature vit sous le régime de la crise depuis bien longtemps, c'està-dire que d'aucuns la proclament en crise voire en péril. Mais ces discours de crise sont devenus un lieu commun chez certains critiques (tels T. Todorov, W. Marx...) et ils se couplent avec une persistante "querelle de l'enseignement littéraire ». Ainsi se redessine, une tension de longue durée entre une conception de la Littérature comme un absolu, qui appelle une lecture immanente et vaut comme inculcation d'un ordre moral, et une conception qui envisage la 
littérature (ou le littéraire) comme une activité associée à d'autres au sein des outils de connaissance et qui incline vers des modes de lecture transférables ré-investissables. Ainsi apparaît-il combien le littéraire est un des espaces culturels où se joue de façon particulièrement aiguë l'enjeu social des attributions de qualifications.

In France, the $21^{\text {st }}$ Century has been a time full of discourses on the idea of literary crisis. Historically speaking, it does not sound particularly new: for ages, Literature was constantly a topic of quarrels in which lot of texts pointed out various crisis. What is new is that some critics (as T. Todorov or W. Marx) make addresses with an argument about «crisis and dead of Literature " and, at the same time, there are quarrels about how to teach Literature. Some argue that it must be an end in itself and promote a immanent reading. In an other hand, some others argue that reading should be a way to access to knowledge without borders. Therefore, it is today quite clear that literacy constitutes a cultural field where the social debate about how qualifications run is specially obvious.

INDEX

Keywords : knowledge, crisis, teaching, immanence, reading, literary, literture, moral Mots-clés : Connaissance, Crise, Enseignement, Immanence, Lecture, Littéraire, Littérature, Morale

\section{AUTEUR}

ALAIN VIALA

Université d'Oxford, OX1 2JD Oxford, Angleterre 